temperature solutions is considered; not only the practice, but also the theory is presented and, last but not least, not only the problems that have been solved, but also those that are to be solved in the near future are dealt with.

High-temperature solution growth is defined to encompass growth from solutions between 300 and $1800^{\circ} \mathrm{C}$, including VLS (vapour-liquid-solid) growth, excluding hydrothermal growth. Several of the methods discussed in the book are applied on an industrial scale to produce single crystals for various purposes: magnetic oxides, semiconductor devices, non-linear optical materials and gemstones.

The book consists of ten chapters. After an introduction there is a chapter on the history of crystal growth from solutions which reads like a thrilling novel. $\mathrm{Ch} .3$ on solvents and solutions considers briefly the thermodynamics and extensively the chemical equilibria and complex formation in solutions. Useful attention is given to the properties of practical solvents. Ch. 4 on nucleation and on the theory of crystal growth is a fine introduction to the subject that could be used separately for a graduate course on crystal growth. Ch. 5 treats quite generally the influence of growth conditions and impurities on crystal habit. It ends with an extensive documentation on six important substances. Ch. 6 deals with morphological stability and closely related subjects such as constitutional supercooling, facetting and solution flow, including temperature and stirring programming. In Ch. 7 (154 pp.) the experimental techniques are extensively described in a systematic way. First, techniques for seeding, for producing supersaturation and for avoiding spontaneous nucleation, then the technology (furnaces, temperature control and programming, crucibles, stirring) and finally some special techniques. Ch. 8 describes separately liquid-phase epitaxy. Ch. 9 deals with problems that arise after the crystals have been grown: characterization (chemical analysis, inhomogeneities, optical methods and $\mathrm{X}$-ray methods, defect determination). It ends with a proposed standard for routine characterization, which would need quite an investment of apparatus, but which would probably pay off in the long run. Ch. 10 gives a table of about 1400 entries with data of HTS-grown crystals.

The literature is almost exhaustively covered, the chapters contain references to many reviews and even older mineralogical literature is not neglected. When a disagreement appears in the literature the authors sometimes give their own opinion, sometimes give both (or more) views. There are instances where a reader would wish to have more guidance from the authors, as, for example, in the discussion on the habit of yttrium garnets (p. 227), where the Tatarsky paper settles the issue. In a few cases the theory appears at unexpected places. For example, it is better not to hide the important problem of distribution coefficients in $\mathrm{Ch} .7$ (techniques), but to place it in either Ch. 3 or 4 . Misprints are very rare and not disturbing. The presentation is very good on the whole, although some reproductions are not clear. The book is worth its price.

In their preface the authors state that 'the book is designed to become the standard reference work in the field of crystal growth from high-temperature solutions'. Will it reach that goal? I think it will.

P. HARTMAN

Geologisch en Mineralogisch Instituut der Rijksuniversiteit Garenmarkt $1 \mathrm{~B}$

Leiden

Netherlands
Growth of crystals, Vol. 9. Edited by N. N. SHEFTAL' and E. I. Givargizov. Pp. $x+329$, Figs. 261, Tables 23. New York and London: Consultants Bureau, 1975. Price $\$ 42.00$.

This ninth volume in the already well-known series Growth of Crystals contains the translation of the Rost Kristallov conference held at Leningrad in May 1969, immediately after the Fedorov memorial meeting. According to N. N. Sheftal's introductory address the scope was restricted principally to the mineralogical aspect of crystal growth, which indeed appears from the fact that most compounds dealt with have crystal structures that occur also in nature.

V. B. Tatarskii starts with a survey of the few papers devoted by $\mathrm{E}$. S. Fedorov to crystal growth phenomena. It is comforting to read how even such a great scientist as Fedorov sometimes went astray in his explanation of observed phenomena.

The papers are divided among six sections: hydrothermal (11 papers) and diamond (3 papers) synthesis, solution growth (3), flux growth (11), melt growth (17), vapour growth (14) and theory (4). An appendix contains a memorial paper by N. N. Sheftal' to Academician A. V. Shubnikov, a paper on the equilibrium shape of potash alum crystals by N. M. Shchagina, one of the last studies made under Shubnikov's guidance, a closely related paper by $N$. N. Sheftal' on the limiting shapes of single crystals of Rochelle salt and potash alum, while finally N. N. Sheftal' pays a tribute to Academician N. V. Belov on the occasion of his 80th birthday.

What to say about these papers seven years after they were presented and four years after they appeared in print? The quality of the papers is rather variable. Many of them just contain data that are still useful, but several lack information on data that must have been available and which information would have added greatly to a better understanding of the reported results. For example, in an interesting paper on the hydrothermal growth of calcite seeds the composition of the medium is not given. Most papers describe extensively the surface morphology of the synthesized crystals, which is as it should be at a Fedorov memorial conference, but measurements are rare. It is interesting to see how some papers were the nuclei of important work that is now in progress or that has been published recently. To mention a few examples: the study by $\mathrm{E}$. I. Givargizov on the VLS growth of Ge and Si whiskers, the morphology of cyclohexanol crystals growing from the melt by D. E. Ovsienko et al. and the growth of diamond by V.P. Butuzov et al. Other papers are still important because of the intriguing results presented, as for example the work by T. G. Petrov on anomalies in the growth rates of $\mathrm{KCl}$ at various temperatures and constant supersaturation.

Every crystal-growth scientist will find something of interest in this volume, but one wonders why it did take so many years to prepare this book, especially since the Russian edition of the tenth Rost Kristallov conference has already appeared. Finally a few specific remarks must be made. The translation seems to be very good, but growth hummocks are usually called growth hillocks. As a result of the double transliteration several names of even well-known non-Russian authors contain spelling errors. Finally, E. D. Dukova is well known for her work on the crystallization of $\beta$-methylnaphthalene, but she never worked on quartz as the title suggests. 
In conclusion, a useful volume that appeared too late.

P. HARTMAN

Geologisch en Mineralogisch Instituut der Rijksuniversiteit Garenmarkt $1 \mathrm{~B}$

Leiden

Netherlands

Anomalous scattering. Edited by S. RAMASESHAN and S. C. Abrahams. Copenhagen: Munksgaard, 1975. Pp. xi + 539. Price D. Kr. 200.00.

In April 1974 an Inter-Congress Conference was held in Madrid on the subject of anomalous scattering. The results of this meeting have been assembled into a welcome and valuable book dealing with every conceivable aspect of anomalous scattering.

The book is organised into eight chapters each dealing with a different general theme and within each chapter individual papers are presented by their authors. An indication of the coverage of the whole work will be given under the chapter headings.

\section{Historical Survey}

This begins with a well-written narrative-style account by A. F. Peerdeman of the discovery of the anomalous scattering of X-rays by Bijvoet and his coworkers. Peerdeman makes an interesting remark 'It was about that time after the first elation over the success of our anomalous child that I had a feeling of slight disappointment because this child appeared to be anomalous in another respect. It seemed to be fully grown at its birth'. This remark seems justified by what follows in the book; the theory and the implications of anomalous scattering were known almost immediately the effect was conceived and all subsequent work has been the refinement of theory, the improvement of measurement techniques and the discovery of new applications.

\section{Experimental Determination of $f^{\prime}$ and $f^{\prime \prime}$}

This chapter includes a number of papers describing the experimental measurements of the real and imaginary components of anomalous scattering for various elements $(\mathrm{Cu}$, Co etc.) and also measurements on simple binary compounds. The agreement between measurement and theory is, in general, very satisfactory.

\section{Novel Applications of Anomalous Scattering}

A great deal of what is contained in this chapter would not normally be associated with anomalous scattering by a conventionally trained X-ray crystallographer - for example, non-kinematic and inelastic scattering of electrons, the identification of atomic environment and excess diffuse $\mathrm{X}$-ray scattering. The structural crystallographer will find interesting the paper by S. Ramaseshan, T. G. Ramesh and G. S. Ranganath on the application of anomalous scattering to centrosymmetric structures where scattering at two different wavelengths gives information similar to that obtained from isomorphous replacement.

\section{Absolute Configuration and Tensorial Properties}

Four of the papers in this chapter are concerned with various aspects of determining absolute configuration including the sign of tensorial properties. The fifth and final paper in this section, concerned with the experimental determination of $f_{\mathrm{Ga}}^{\prime \prime}$ for Mo $K \alpha$, seems more properly to belong to Chapter II.

\section{Accurate Intensity Measurement, Including Anomalous Scattering}

When the idea of looking for Bijvoet differences was first mooted, many researchers believed that the differences of intensity of Friedel pairs of reflexions would be too small to observe. Indeed, in the foreword, Professor Dorothy Hodgkin freely admits that she was among their number. While this conclusion was incorrect what is true is that the information to be gained from anomalous scattering can depend critically on the precision of intensity measurements. The four papers contained in this chapter are full of interest to the structural crystallographer with theory, experiment and the analysis of results all being included.

\section{Effects of Dispersion on Refined Atomic Parameters}

The chapter begins with two papers on errors in atomic positions due to neglect of the anomalous component of scattering. Once again the third and final paper seems out of place. This paper is by P. J. Black and R. N. Corby on the application of anomalous scattering to the solution of crystal structures and includes ideas on linking anomalous scattering to direct methods of solving structures.

\section{Anomalous Scattering and Structure Analysis of Macromolecules}

Over 160 pages are devoted to this most important topic. The two initial papers, which deal with the general techniques of applying anomalous scattering to macromolecules, have a heavy overlap in content. Other papers deal with specific applications. The final paper of this chapter is by Mössbauer on the application of anomalous dispersion of $y$ radiation.

\section{Structure Determination Using Neutron Anomalous Scattering}

Some atomic nuclei give anomalous scattering of thermal neutrons and this has been exploited over the past few years. There are only two papers in this section but they are important ones and may point the way to a powerful method of solving the phase problem when suitable nuclei are present in the structure.

There is a brief Discussion section associated with the material of each chapter and some of the points raised therein add greatly to the usefulness of the book.

With so many authors one cannot usefully comment on the quality of writing and presentation. However the editors have done their work well and the whole work hangs together as an entity.

The book is marred by a number of errors, some fairly significant, but current sales of the book are accompanied by an errata sheet which undoes most of the damage.

This is a valuable contribution to crystallographic literature and all working crystallographers should have access to a copy.

Department of Physics

M. M. WOOLFSON

University of York

Heslington

York YO1 5DD

England 